\title{
How to measure the Vitamin-D-synthetic activity of UV lamps used in phototherapy?
}

\author{
Irina P Terenetskaya* \\ Doctor of Phys.-Math. Sciences, Professor, Leading Scientist, Department of Optical Quantum Electronics, Institute of Physics NAS, Ukraine
}

\begin{abstract}
Ultraviolet lamps are widely used in phototherapy, and the positive effect of ultraviolet radiation is mainly associated with the synthesis of vitamin D in human skin. Nevertheless, to avoid harmful effects the biological efficacy of UV lamps is still evaluated based on the erythema action spectrum using UV detectors with an output in erythema units. Evaluation of vitamin D synthesis on this basis is inadequate because of the difference between the erythema and the Vitamin $\mathrm{D}$ synthesis action spectra. Hence, direct measurement of the vitamin-D-synthetic activity is a missing link in the metrology of UV lamps that are used for medical and/or cosmetic purposes. This paper presents original methods based on the same photoreaction in vitro by which vitamin $\mathrm{D}$ is synthesized in human skin via photo- and thermo- induced conversions of 7-Dehydrocholesterol (provitamin $\mathrm{D}_{3}$ ). The UV photons are absorbed by provitamin $\mathrm{D}$ molecules in solution or embedded in specially designed UV transparent and stable matrix mimicking biological samples. Three operation modes of varying complexity have been developed to follow the photoreaction course in real time, and the results are presented of measuring the vitamin D synthesizing activity of several UV lamps, as well as the first performed comparative studies on direct measurements of the vitamin $\mathrm{D}$ level in blood and in solution.
\end{abstract}

\section{Introduction}

The discovery of UV radiation in the $19^{\text {th }}$ century, its properties and the connection with physiological and pathological changes in humans led to the conclusion that UV radiation has both beneficial and harmful effects, depending on the type of organism, wavelength region and the radiation dose.

The varied effects derive, in part, from the differences among UV-A (320-400 nm), UV-B (280-320 nm) and UV-C (100-280 nm) photons energy and also due to the different structures that are capable of absorbing UV photons in living organisms. UV photons are absorbed by UV sensitive molecules in skin and initiate a variety of photochemical reactions resulting in structural changes that could lead to positive or negative biologic effects depending on the accepted UV dose.

Deoxyribonucleic acid (DNA) is one of the most important target molecules for photobiological effects. A large number of different types of UV induced photoreactions are produced in DNA molecule that can result in mutations and even cell lethality. Formation of cyclobutylpyrimidine dimers in DNA contributes to the mechanism of erythema and sunburn formation [1]. Fortunately, a cell has an enormous capacity to repair all types of damage to its DNA.

Another chromophore in the epidermis is 7-dehydrocholesterol (7DHC, provitamin $\mathrm{D}_{3}$ ), and the UVB photons penetrating into human skin are responsible for its conversion into the active form of vitamin $\mathrm{D}_{3}$, necessary for the normal calcium absorption and metabolism in the body. In recent years interest in Vitamin D has increased greatly in view of new data about its important role in reducing the risk of cancer, multiple sclerosis, and type 1 diabetes mellitus [2]. The vitamin D active metabolite $1,25(\mathrm{OH})_{2} \mathrm{D}_{3}$ is recognized as a critical hormone regulating cell growth and modulating the immune system [3].

The measurement of the vitamin-D-synthetic ability of UV lamps used for medical and/or cosmetic purposes is especially important given the essential role of vitamin D in maintaining health, as well as taking into account the observed pandemic of vitamin $\mathrm{D}$ deficiency among the world's population [2].

In this article we will focus on the calculation and measurement of the vitamin D effective irradiance of UV sources using original method based on an in vitro model of vitamin D synthesis.

\section{Action spectra and biologically effective irradiances}

Each specific biological effect of UV radiation is characterized by its own action spectrum (AS) defined as the spectral dependence of the value of the biological effect initiated by monochromatic radiation of different wavelength with the same dose. A biologically effective irradiance $\boldsymbol{E}_{\text {eff }}$ of a UV source can be calculated by weighting its spectral irradiance by the appropriate action spectrum and integrating over the wavelength interval for which the action spectrum is non-zero [4].

$$
E_{\text {eff }}=\int E_{\lambda}(\lambda) * S_{\lambda}(\lambda) d \lambda
$$

Here $E_{\lambda}(\lambda)$ - spectral irradiance of a UV lamp measured by a spectroradiometer $\left[\mathrm{Wm}^{-2} \mathrm{~nm}^{-1}\right], S_{\lambda}(\lambda)$ - action spectrum [relative units], $\lambda$ - wavelength $[\mathrm{nm}]$.

Being mathematically determined, the CIE erythema action spectrum is widely used for estimation of the erythemally active irradiance of sunlight and artificial UV sources [5].

Correspondence to: Irina P Terenetskaya, Doctor of Phys.-Math. Sciences, Professor, Leading Scientist, Department of Optical Quantum Electronics, Institute of Physics NAS, Ukraine, E-mail: teren@iop.kiev.ua

Key words: UV radiation, UV biodosimeter, Vitamin D synthesis, antirachitic UV dose

Received: April 08, 2018; Accepted: April 27, 2018; Published: April 29, 2018 
The situation with the Vitamin-D synthesis action spectrum is more difficult and ambiguous. In 2006 CIE proposed action spectrum for the production of previtamin $\mathrm{D}_{3}$ in human skin [6] based on the single measurement [7] made before the important wavelength effect was discovered due to UV tunable laser initiation [8]. This little-known wavelength effect is described in detail in [9].

As is known, the synthesis of vitamin $\mathrm{D}$ is a two-step process. First, the UV photon absorption leads to the conversion of 7-DHC into previtamin $\mathrm{D}$, which subsequently undergoes a thermo-induced conversion into vitamin D. Therefore, the amount of previtamin D accumulated during an UV exposure is a measure of a biologically effective ('antirachitic') UV dose.

Since the rate of previtamin D accumulation at the initial stage of UV irradiation is primarily dependent on the efficiency of photon absorption by 7-DHC molecule, the action spectrum of vitamin $\mathrm{D}$ synthesis must correlate with the absorption spectrum of 7-DHC. However, such correlation of the 7-DHC absorption spectrum with the in vivo AS measured in [7] is absent, although a close coincidence of the formation of previtamin $\mathrm{D}_{3}$ from 7-DHC in both the human epidermis and the solution was found in the same work.

A detailed analysis of the reasons for this discrepancy is given in [10]. Some doubts whether the CIE action spectrum is correct are also discussed in $[11,12]$. Such uncertainty in the Vitamin D action spectrum could significantly alter the interpretation of the vitamin D synthetic capacity of a UV source.

Therefore, taking into account that photobiological effects are initiated by photochemistry, it seems more reasonable to use the absorption spectrum of 7-DHC in 'weighted spectroradiometry' instead of the CIE Vitamin D action spectrum in human epidermis to avoid misleading results in the forecasting the vitamin-D-effective irradiance of UV lamps. It should be emphasized that this approach is fully consistent with the first law of photochemistry - 'Light must be absorbed for photochemistry to occur?. It should also be mentioned that the measurements of the Vitamin D action spectrum in vitro revealed its close correspondence to the absorption spectrum of 7-DHC [13].

It is important to realize that erythema AS is not suitable for the calculation of 'antirachitic' irradiance [14] as illustrated in Figure 1, where two action spectra (erythema and Vitamin D) are shown together with the UV lamp spectrum (Figure 1a) and the corresponding calculated biologically effective irradiances are presented in Figure 1b. It is clear that antirachitic irradiance is a small part of the erythema irradiance for this UV lamp. In other words, the number of UV photons initiating the synthesis of previtamin $\mathrm{D}$ is significantly less than the number of UV photons which can cause a burn.
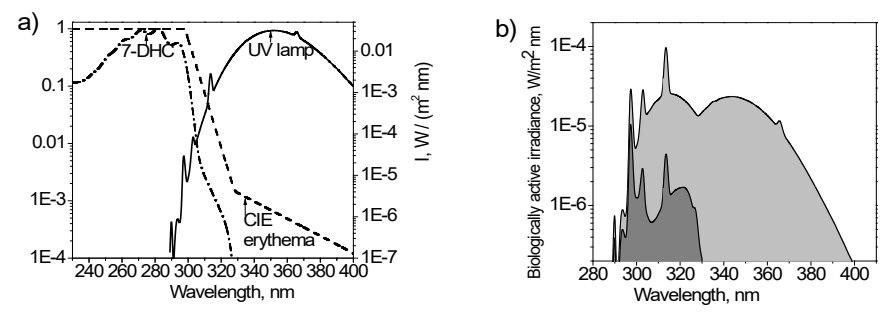

Figure 1. The absorption spectrum of 7-dehydrocholesterol (relative units) and CIE erythema action spectrum in relation to the irradiation spectrum of the UV lamp Cleoperformance 100W-R (a) and calculated erythemic (light grey) and 'antirachitic' (dark grey) biologically active irradiances of the lamp (right)
It should also be emphasized that the ratio of erythemic and antirachitic irradiance is not constant and depends on the emission spectrum of a particular UV lamp.

Another way to directly measure the biological effectiveness of $\mathrm{UV}$ radiation is to use special instrument whose spectral sensitivity correlates with the action spectrum of a particular biological effect. Most of broadband UV detectors are commonly designed to have a spectral sensitivity which is a close match to the CIE erythema action spectrum. As follows from Figure 1 such instruments cannot adequately measure the Vitamin-D-synthetic ('antirachitic') activity of a UV source. This problem can be solved with the help of special biodosimeter which measures integrated biological effect directly, but the result is expressed in specific biological units.

For direct checkout of the vitamin D synthetic capacity of a UV source the bio-equivalent UV dosimeter has been developed that is based on the same molecular photochemistry from which vitamin $\mathrm{D}$ is photosynthesized in human skin and makes possible both instrumental [15-18] and visual indication of vitamin D synthesis [19-21].

\section{Main features of vitamin D synthesis}

As mentioned above, the production of vitamin $\mathrm{D}$ is a two stage process. The first step is photoinduced formation of previtamin $\mathrm{D}$ from provitamin $\mathrm{D}$ as a result of UV photons absorption. The second step is thermoinduced conversion of previtamin D into vitamin D (Figure 2). Therefore to determine the antirachitic UV dose it is required to measure the amount of previtamin D accumulated during UV exposure, but the task is complicated by the fact that previtamin D itself is not stable to UV radiation and undergoes a number of side photoconversions.

As a result, UV irradiation of initial provitamin $\mathrm{D}$ gives rise to the formation of a multicomponent photoisomer mixture in which the maximum amount of accumulated previtamin D significantly depends on the wavelength of UV irradiation (or on the spectral composition of a UV lamp radiation) (Figure 3)

Another important feature of the photoreaction is non-linear dependence of previtamin D accumulation on a UV dose, that is, it is incorrect to believe that the longer the UV exposure, the more previtamin $\mathrm{D}$ is formed.

Hence any measuring device (optoelectronic or a photosensitive polymer film) with a linear response cannot adequately measure the vitamin-D-synthetic activity, even if its spectral sensitivity corresponds to the action spectrum of vitamin D synthesis. A suitable measuring device should also have the same nonlinear response to the dose of UV radiation. Obviously, the best tool for this measurement is an in vitro model of vitamin D synthesis.

\section{Methods}

For the first time in vitro model of vitamin D synthesis (cylindrical ampoule with ethanol solution of 7-Dehydrocholesterol) was used to clarify the effect of seasonal and latitudinal changes in solar UVB radiation on vitamin $\mathrm{D}_{3}$ synthesis [22]. In this study the concentration of accumulated previtamin $\mathrm{D}$ was determined using high-performance liquid chromatography (HPLC) which is not suitable for UV measurements in situ.

A significant steps towards the widespread practical use of the in vitro model for on-site measurements were exposure of the ethanol solution of 7-DHC in a standard rectangular spectrophotometric cuvette and the development of original spectrophotometric analysis 


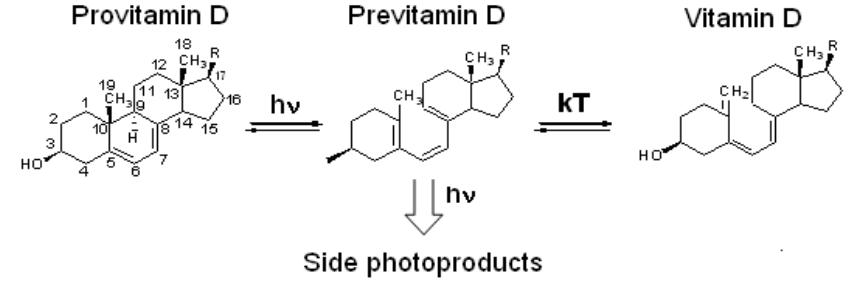

Figure 2. Schematic representation of vitamin D synthesis: UV irradiation of provitamin $\mathrm{D}$ yields previtamin $\mathrm{D}$ which is further converted into vitamin $\mathrm{D}$ at body temperature, but upon UV irradiation, the newly formed previtamin D is subject to side photoconversions
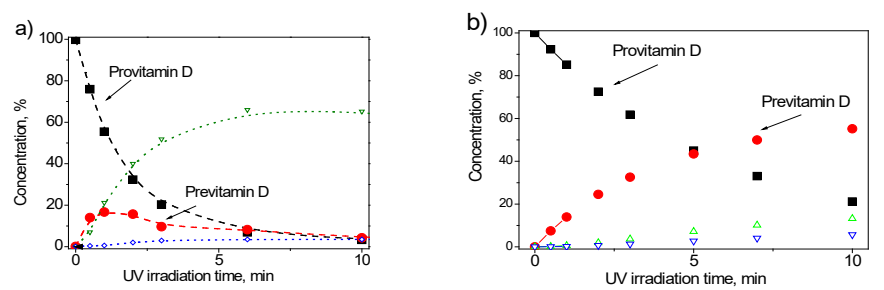

Figure 3. The course of the photoreaction of previtamin D formation from 7-DHC under UV irradiation (a) by the low pressure mercury lamp (254 nm) and (b) by the XeCl excimer lamp $(308 \mathrm{~nm})$. Open symbols denote side photoproducts

of the photoisomer mixture formed under UV irradiation of 7-Dehydrocholesterol [17].

Moreover, owing to the detailed studies of the photoreaction using a tunable dye-laser [8] the adequate mathematical model was developed enabling calculation of the photoreaction kinetics for any UV radiation source. The validity of spectral analysis and the mathematical model was confirmed by simultaneous UV measurements using a spectroradiometer within the framework of the EC BIODOS Project [16].

To date, there are three possible measurement methods based on an in vitro model of vitamin $\mathrm{D}$ synthesis in which the targets for UV photons are 7-DHC molecules either in solution or embedded in specially designed UV transparent and stable matrix.

1. Measurement of Previtamin D concentration using spectrophotometric analysis: a cuvette with ethanol solution of 7-DHC is irradiated by a UV source, the absorption spectra are recorded before irradiation and after several exposures, and further for concentration analysis the spectra are processed by computer using original software [15-17].

2. Measurement of Previtamin D concentration using calibration graph: the transparency changes of the film (polymeric or hydrogel) with embedded 7-DHC molecules before and after a UV exposure are measured at a specific wavelength by a spectrophotometer or using specially developed portable optoelectronic device 'VitaD', and previtamin $\mathrm{D}$ concentration is determined by comparison with calibration graph [18].

3. Visual detection of the antirachitic dose by dissolving 7- DHC in liquid crystal; first by changing the number of the Cano-Grangean bands in wedge LC cell [19], then by changing the color of a LC mixture in a plane-parallel cell (adding a twisting additive) [20], and finally by turning the disclination line in the LC theta-cell [21].

We will not give more details here, since these three methods with all references are described in detail in recent publication [23].

\section{Results and discussion}

A number of laboratory tests were carried out using various UV sources, the radiation of each was measured by a spectroradiometer. Here we present the results of measurements of UV lamps relevant for medical applications as well as the result of the biomedical research, establishing a link between the measurements in vitro and in vivo.

Verification of the reciprocity law was carried out by irradiation of the cuvette with ethanol solution of 7-DHC with standard lamp BBH4 (1000W FEL, Osram Sylvania Ltd) which is commonly used for spectroradiometer calibration [24]. According to the rule for a point light source it was found that doubling the distance between the lamp and the cuvette led to a four-fold increase of the exposure time to get the same concentration of previtamin D.

A similar result was obtained with the UV arc discharge mercury lamp of high pressure (DRT-125-1) which is used in many household appliances of UV irradiation [25]. The accumulation of previtamin $\mathrm{D}$ in vitro was measured at two distances $(90$ and $180 \mathrm{~cm})$ and the corresponding concentration dependences are shown in Figure 4. It should be noted that the amount of previtamin $\mathrm{D}$ accumulated by the 10th minute in the 1st case and by the 40th minute in the 2nd case is approximately equal.

As can be seen from Figure $4 \mathrm{~b}$ ), the previtamin $\mathrm{D}$ accumulation can be approximated by a linear dependence only with short exposures (up to $10 \mathrm{~min}$ ). With further irradiation, the curve goes to the saturation mainly due to the side cis-trans photoisomerization of previtamin D.

In addition, the vitamin-D synthesizing power was compared for the two lamps (The results of measurements of these two lamps were kindly provided by Dr. Tetiana Orlova) (Philips TL 20W / 01 RS SLV and Philips TL 20W / 12 RS SLV) recommended for the treatment of psoriasis. Their spectra measured by a spectroiradiometer are shown in Figure 5, and Figure 6 shows the measured kinetics of the photoreaction in vitro. a)

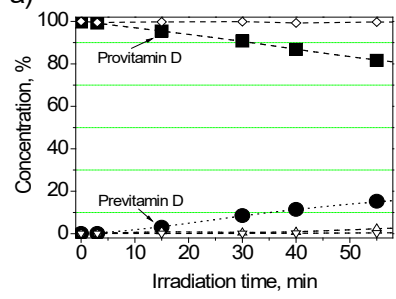

b)

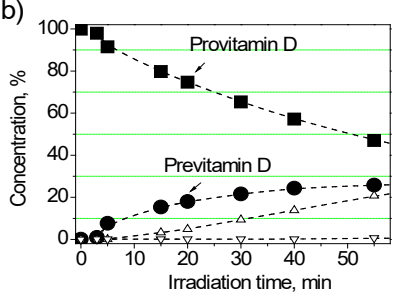

Figure 4. Change in the concentration of the initial 7-DHC (squares) and formed previtamin D (circles) under irradiation by the lamp DRT-125-1 at the distances of 180 (a) and $90 \mathrm{~cm}$ (b)

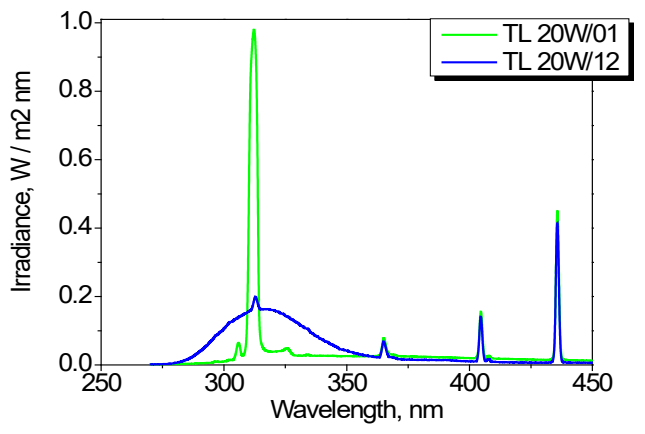

Figure 5. UV spectra of the two UV lamps for the treatment of psoriasis 

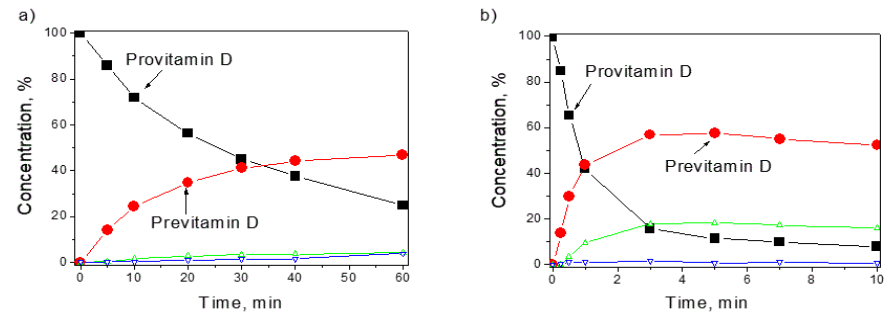

Figure 6. Concentration dependences upon irradiation of 7-DHC in vitro by the two UV lamps TL01 (a) and TL12 (b)

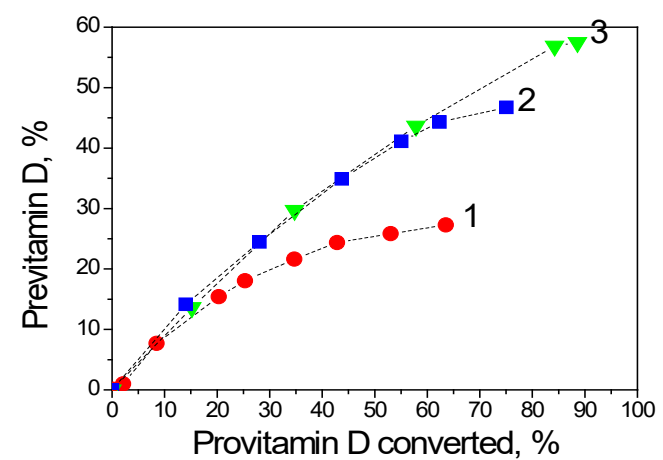

Figure 7. Comparative dependencies of previtamin $\mathrm{D}$ photosynthesis relative to converted provitamin D for three lamps: 1 - DRT-125-1, 2 - Philips TL 20W / 01 RS SLV, 3 - Philips TL 20W / 12 RS SLV

Comparative vitamin D synthesizing activity of three lamps is given in Figure 7 where formation of previtamin D is shown in relation to the converted provitamin D. One can conclude that, the Philips TL lamps are more favorable for the synthesis of previtamin $\mathrm{D}$ than the high pressure mercury lamp, and the broadband lamp Philips TL12 is much better than the narrowband TL 01 , since $60 \%$ of the previtamin is produced at $90 \%$ conversion of initial provitamin D.

Finally, we will touch upon a single study that was conducted to provide a missing link between the in vivo and in vitro measurements in which the $25(\mathrm{OH}) \mathrm{D}$ level (After its formation in skin vitamin D3 is metabolized to 25 -hydroxyvitamin $\mathrm{D}(25(\mathrm{OH}) \mathrm{D})$ in liver [3]) in the blood of healthy volunteers exposed to artificial UV source was compared with the accumulation of previtamin D in solution [26]. The UV source was a commercially available and approved sunbed Wolff Suveren 53IG (Wolff System, Basel, Switzerland).

The volunteers were divided into three groups based on the results of $25(\mathrm{OH}) \mathrm{D}$ measurements at the start of the investigation: with insufficient levels $(25-50 \mathrm{nmol} / \mathrm{L})$, marginal $(50-75 \mathrm{nmol} / \mathrm{L})$ and sufficient levels $(75-150 \mathrm{nmol} / \mathrm{L})$ of $25(\mathrm{OH}) \mathrm{D}$ in blood. The study lasted 10 weeks. Each participant was exposed 2 times per week during irradiation time $10 \mathrm{~min}$ corresponded to erythema dose $0.74 \mathrm{MED}$ (or $185 \mathrm{~J} / \mathrm{m} 2$, or $1.85 \mathrm{SED}$ ) in conditions of whole body exposure.

To measure the concentration of formed previtaminD in vitro, the solutions of $7 \mathrm{DHC}$ in ethanol $\left(\mathrm{C}=(2.5 \pm 0.3) \cdot 10^{-5} \mathrm{~mol} / \mathrm{L}\right)$ in a rectangular quartz cuvettes $(\mathrm{d}=1 \mathrm{~cm})$ were exposed inside the sunbed with the same total exposure.

It was found that the increase in $25(\mathrm{OH}) \mathrm{D}$ concentration depended both on the initial $25(\mathrm{OH}) \mathrm{D}$ level and on the cumulative sunbed exposure time. It is noteworthy, that the non-linear character of $25(\mathrm{OH}) \mathrm{D}$ formation was observed in vivo, i.e. maximal 25(OH)D levels for all the groups were achieved after 15 sunbed sessions and further
UV radiation up to 20 sunbed sessions almost did not increase $25(\mathrm{OH})$ $\mathrm{D}$ level and thus had no effect on vitamin D status.

Since such behavior was similar to non-linear previtamin D accumulation in vitro, a linear correlation with high correlation coefficients $\mathrm{R}$ has been revealed between the in vivo and in vitro data for the three groups of volunteers with different initial 25(OH)D levels (Figure 8).

It is important to note that observed linear correlations between in vivo and in vitro data allow predicting the changes of vitamin D status after UV exposures using only one pre-exposure blood sample analysis combined with further measurements of previtamin $\mathrm{D}$ accumulation in vitro. Certainly, correlations obtained in this study should be remeasured for each sunbed equipped with other fluorescent lamps.

\section{Conclusions}

Wide use of UV radiation in medicine requires reliable control because high energy UV photons initiate a variety of photochemical reactions that have both negative and positive consequences.

Little attention is still given to the determination of the lowest healthy UV doses that are extremely necessary to overcome the vitamin $\mathrm{D}$ deficiency by maintaining optimal levels of 25 -hydroxyvitamin $\mathrm{D}$ in the human blood. This is especially important in view of its role against many types of cancers, in regulating cell growth and modulating the immune system [3].

In view of strong wavelength dependence of vitamin D synthesis, the specification of artificial UV radiation in radiometric units is of limited value if the spectral content is not taken into account. Most of broad-band UV detectors measuring erythemal activity of UV lamps are unsuitable for determining the specific antirachitic UV exposures.

It is believed that the results given in this article confirm the ability of the presented methods to provide a reliable measure of the antirachitic radiation of UV sources and will form a basis for the future mandatory metrology of the vitamin D synthetic activity of UV lamps used both for medical and cosmetic purposes.

\section{Acknowledgments}

This work was supported by the EU (Grant No.IC20-CT96-0026) and by the Science and Technology Center in Ukraine (Projects Gr50 and P344). The work of Dr. Tetiana Orlova at the Oslo University Hospital was supported by The Research Council of Norway, Yggdrasyl programme, Personal mobility Grant 202615.

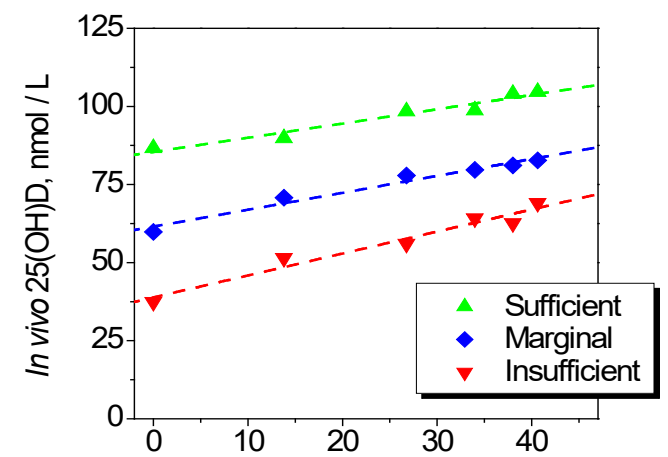

Figure 8. Linear correlations between in vivo changes of $25(\mathrm{OH}) \mathrm{D}$ distribution median in three volunteer groups and in vitro photosynthesis of pre-vitamin D 


\section{References}

1. Kochevar I (1991) Acute effects of ultraviolet radiation in skin. In: M.Holick \& A.Klingen Eds. 1992. Biologic effects of light. Walter de Gruyter, Berlin, New York.

2. Holick MF (2004) Vitamin D: Importance in the prevention of cancers, type 1 diabetes, heart disease and osteoporosis. American J Clinical Nut 79: 362-371. [Crossref]

3. Mayer AC, Norman AW (1991) Vitamin D. In: Encyclopedia of Human Biology, 7, New York: Academic Press. pp.859-871.

4. Horneck G (1995) Quantification of the biological effectiveness of environmental UV radiation. J Photochem Photobiol B: Biology 31: 43-49.

5. McKinlay AF, Diffey BI (1987) A reference action spectrum for ultraviolet induced erythema in human skin. CIE $J$ 6: 17-22.

6. Bouillon R, Eisman J, Garabedian M, Holick M, Kleinschmidt J, et al. (2006) Action spectrum for the production of previtamin D3 in human skin. CIE J 174: 1-12.

7. MacLaughlin JA, Anderson RR, Holick MF (1982) Spectral character of sunlight modulates photosynthesis of previtamin $\mathrm{D}_{3}$ and its photoisomers in human skin. Science 216: 1001-1003. [Crossref]

8. Gundorov SI, Davydenko VA, Terenetskaya IP, Yuschuk OI (1991) Study of the kinetics and quantum efficiencies in the photochemistry of provitamin D using a tunable laser. Sov J Quantum Electronics 21: 339-343.

9. Terenetskaya I (2011) The Little-Known Wavelength Effect in the Provitamin D Photochemistry: The Ambiguous Role of Weak Irreversible Channel", In: Photochemistry: UV/ VIS spectroscopy, Photochemical Reactions and Photosynthesis, Nova Science Publishers.

10. Terenetskaya I (2014) Mismatch between the in vivo and in vitro vitamin D synthesis action spectra: cause-and-effect relationship. CIE DR 6-41 Technical report 2014: 04

11. McKenzie RL, Liley JB, Bjorn LO (2009) UV radiation: balancing risks and benefits. Photochemistry and Photobiology 85: 88-98. [Crossref]

12. Norval M, Bjorn LO, Gruijl FRD (2009) Is the action spectrum for UV-induced production of previtamin $\mathrm{D}_{3}$ in human skin correct? Photochem Photobiol Sci 9: 11-17. [Crossref]

13. Bolsée D, Webb AR, Gillotay D, Dörschel B, Knuschke P, et al. (2000) Laboratory facilities and recommendations for the characterization of biological ultraviole dosimeters. Appl Opt 39: 2813-2822. [Crossref]
14. Terenetskaya I (2003) Duality of solar UV-B radiation and relevant dosimetry: vitamin D synthesis versus skin erytema, In: Ultraviolet Ground and Space-based measurements, Models and Effects.II, Eds Slusser JR, Herman JR and Gao W., SPIE, 4896, pp.144-150.

15. Terenetskaya I (1994) Provitamin D photoisomerization as possible UVB monitor: kinetic study using tunable dye-laser. Proc SPIE 2134B: 135-140.

16. Galkin ON, Terenetskaya IP (1999) Vitamin D biodosimeter: basic characteristics and potential applications. J Photochem Photobiol B 53: 12-19. [Crossref]

17. Terenetskaya I (2000) Spectral monitoring of biologically active solar UVB radiation using an in vitro model of vitamin D synthesis. Talanta 53: 195-203. [Crossref]

18. Terenetska IP, Orlova TM, Kirilenko EK, Galich GA, Eremenko AM (2013) A method for In Situ determination of the vitamin-D-synthetic dose of natural and artificial ultraviolet irradiation and its implementation in a personal bio-dosimeter. US Patent 8552391 B2.

19. Terenetskaya I (2006) Method of UV dosimetry and device for visual detection of bioactive UVB radiation, Ukrainian Patent № u 200600162 (Priority Date 06.01.06.).

20. Orlova T, Terenetskaya I (2008) UV-biosensor for visual indication of vitamin D synthesis. Proc SPIE 7003: 70031O-1 -70031O-8.

21. Kapinos P, Orlova T, Terenetskaya I (2015) UV biodosimeter with visual detection of vitamin D synthesis using $\theta$-cell. Mol Cryst Liq Cryst 615: 1-8.

22. Webb AR, Kline LW, Holick MF (1988) Influence of of season and latitude on the cutaneous synthesis of vitamin of D3: exposure to winter sunlight in of Boston of and of Edmonton of will not promote vitamin of D3 of in human skin. J Clin Endocrinol Metabol 67: 373-378. [Crossref]

23. Terenetskaya I (2016) Three operation modes of the Vitamin D Biodosimeter, Proc. of SPIE, Vol.9887 Biophotonics: Photonic solutions for better health care V, edited by Jürgen Popp, Valery V. Tuchin, Dennis L. Matthews, Francesco Saverio Pavone.

24. Webb AR, Terenetskaya IP (1998) Combined study of antirachitic solar UVB radiation by spectroradiometer and "Vitamin D" biodosimeter", In: Biologic Effects of Light, Proc.of Int. Conf., 1-3 November, 1998, Basel, Switzerland. / Editors, Michael F. Holick and Ernst G. Jung, Kluver Acad. Publishers, 1999, pp.153-155.

25. Kapinos P, Orlova T, Terenetskaya I (2012) Method for the measurement of the vitamin-D synthetic capacity of UV radiation. Lighting engineering and power engineering 1: 25-33.

26. Orlova T, Moan J, Lagunova Z, Aksnes L, Terenetskaya I, et al. (2013) Increase in serum 25-hydroxyvitamin-D3 in humans after sunbed exposures compared to previtamin D3 synthesis in vitro. J Photochem Photobiol B 122: 32-36. [Crossref]

Copyright: (C2018 Terenetskaya IP. This is an open-access article distributed under the terms of the Creative Commons Attribution License, which permits unrestricted use, distribution, and reproduction in any medium, provided the original author and source are credited. 